\title{
ACE inhibitors and COVID-19: we don't know yet
}

\author{
Taqua Khashkhusha ${ }^{1}$, Jeffrey Chan $^{2}$, and Amer Harky ${ }^{3}$ \\ ${ }^{1}$ University of Liverpool \\ ${ }^{2}$ The Chinese University of Hong Kong \\ ${ }^{3}$ Liverpool Heart and Chest Hospital NHS Trust
}

April 28, 2020

\begin{abstract}
The SARS-CoV-2, the causative agent of COVID-19, has been established to gain access to the human cell via the ACE2 receptor similar to its familial coronavirus SARS-CoV which led to the outbreak in 2003. A concern with the newer 2019 coronavirus is its 10-20-fold higher affinity to the ACE2 receptor that of SARS-CoV, aiding its effective human-to-human transmission which has led to this pandemic. ACE2 receptor expression is thought to be upregulated in use with ACE inhibitors. As ACE inhibitors are known to be a used extensively in the treatment of hypertension it was a concern regarding the risk of using these medications alongside a SARS-COV-2 infection. ACE inhibitors are also used in the treatment regime of other common conditions including diabetes and Cardiovascular disease (CVD). It is worth noting that ACE2 expression has found to be upregulated by the use of thiazolidinediones and ibuprofen too. Consequently, the increased expression of ACE2 would facilitate infection with COVID19. Therefore, it would hypothesise that diabetes and hypertension treatment with ACE2-stimulating drugs would increase the risk of developing severe and fatal COVID-19.
\end{abstract}

ACE inhibitors and COVID-19: we don't know yet

Running Head: COVID-19 and ACEi

Taqua R Khashkhusha1, Jeffrey Shi Kai Chan, MBChB2,3, Amer Harky, MBChB, MRCS, MSc4*

1 School of Medicine, University of Liverpool, Liverpool, United Kingdom 2 Faculty of Medicine, The Chinese University of Hong Kong, Shatin, New Territories, Hong Kong 3 Division of Cardiology, Department of Medicine and Therapeutics, Prince of Wales Hospital, Shatin, New Territories, Hong Kong 4 Department of Cardiothoracic Surgery, Liverpool Heart and Chest Hospital, Liverpool, United Kingdom

* Corresponding author:

Amer Harky

MBChB, MRCS, MSc

Department of Cardiothoracic Surgery

Liverpool Heart and Chest Hospital

Thomas Drive, Liverpool

L14 3PE

United Kingdom

E-mail: aaharky@gmail.com

Phone number: 01516001616 
Key words: COVID-19, ACEi, Corona Virus

Abstract:

The SARS-CoV-2, the causative agent of COVID-19, has been established to gain access to the human cell via the ACE2 receptor similar to its familial coronavirus SARS-CoV which led to the outbreak in 2003. A concern with the newer 2019 coronavirus is its 10-20-fold higher affinity to the ACE2 receptor that of SARS$\mathrm{CoV}$, aiding its effective human-to-human transmission which has led to this pandemic. ACE2 receptor expression is thought to be upregulated in use with ACE inhibitors. As ACE inhibitors are known to be a used extensively in the treatment of hypertension it was a concern regarding the risk of using these medications alongside a SARS-COV-2 infection. ACE inhibitors are also used in the treatment regime of other common conditions including diabetes and Cardiovascular disease (CVD). It is worth noting that ACE2 expression has found to be upregulated by the use of thiazolidinediones and ibuprofen too. Consequently, the increased expression of ACE2 would facilitate infection with COVID-19. Therefore, it would hypothesise that diabetes and hypertension treatment with ACE2-stimulating drugs would increase the risk of developing severe and fatal COVID-19.

Dear Editor,

The recent Chinese clinical studies detailing the clinical characteristics of patients infected by the novel COVID-19 infection have confirmed many of these concerns ${ }^{(1)}$. A study included 1099 patient's laboratoryconfirmed Covid-19 from 552 hospitals in 30 provinces, autonomous regions, and municipalities in mainland China through January 29, 2020, of which 261 had an associated comorbidity ${ }^{(2)}$. Hypertension yet again was the most common comorbidity with 165 patients, followed by 81 patients with diabetes. A meta-analysis (3) evaluating the comorbidities associated with COVID19 found similar results, however noted that in comparison to the prevalence of both diabetes and hypertension in china, the percentage of each comorbidity presenting with COVID19 was similar. These statistics raise the question of whether hypertension itself a high-risk comorbidity is or is the use of ACEI specifically as treatment responsible for these statistics.

Coronary heart disease (CHD) being the most common chronic condition worldwide, a small percentage of COVID-19 patients had the disease as a comorbidity. The lower rates of CHD could be associated with the lower ACE2 receptor expression found to be supressed in patients with the development of heart failure ${ }^{(4)}$. Lower ACE2 expression then, would reduce the ability of the COVID-19 virus to bind and infect the cell. Large cohort studies factoring in ACE2 expression as a variable while comparing progression of COVID19 infection in patients would indicate the relevance of the ACE2 receptor in COVID19 mortality and fatality.

According to data from the above study, $23 \cdot 7 \%$ of patients with hypertension were assessed to have a severe COVID19 infection, followed by diabetes mellitus (16.2\%), coronary heart diseases $(5 \cdot 8 \%)$, and cerebrovascular disease $(2.3 \%)$. A high percentage of $35.9 \%$ of who had hypertension had an end point at ICU, with the use of mechanical ventilation or death. $26.9 \%$ of diabetic patients had a similar end point. As ACE inhibiters are dominantly used in hypertension this could potentially explain the high percentage of COVID19 positive patients who develop severe infection. Diabetic patients could also be on ACE inhibitors slowing down the progression of the vascular complications associated with diabetes hence, the high percentage of diabetic patients developing a severe infection. Still, however much less than hypertension which could be due to the lower use of ACE inhibitors in diabetes in comparison. It has been shown that contracting COVID19 is equal in both hypertensive and diabetic, however hypertensive patients are likely to develop a severe infection in comparison.

On the other hand, studies ${ }^{(5)}$ have suggested the use of ACE inhibitor might be protective against respiratory complications. The binding of SARS-CoV-2 to ACE2 exhausts ACE2, leading to an imbalance of the reninangiotensin-aldosterone system which spirals into acute severe pneumonia. Blocking the renin-angiotensinaldosterone system by ACEI might therefore reduce inflammation in COVID-19 pneumonia, potentially reducing mortality. A recent study ${ }^{(5)}$ compared inflammatory marker found in COVID19 positive patients on ACE inhibitors versus non-ACE inhibitors. It was found that Interleukin 6 a pro-inflammatory marker levels were reduced in the ACEI group. Large studies are needed to delineate the role of ACEI in treating 
COVID-19, ideally both in patients naïve to ACEI, and chronic users of ACEI. Since small centres may have difficulty amassing enough cases, inter-institutional collaborations should be strongly encouraged. These would indicate whether the use of ACE inhibitors in COVID-19 positive cause more harm than good or vice versa which would shine a light on the use of hypertensive medication in these patients.

In short, both the concerns regarding ACE inhibitor use predisposing to infection by SARS-CoV-2 and the idea that ACEI may help treat COVID-19 have valid theoretical bases. At this point, there is insufficient clinical evidence pointing to either being true, further studies are urgently required. Given the known, significant cardiovascular benefits of ACEI, patients should not stop taking them over the above theoretical concerns. These guidelines nonetheless are due to lack of evidence for the use of ACE inhibiters in COVID19 positive patients. Medical workers and researchers worldwide are strongly encouraged to report any available data regarding the relationship between ACEI and COVID-19.

The authors wish to disclose no conflict of interest.

\section{References}

1. Yan R, Zhang Y, Li Y, Xia L, Guo Y, Zhou Q. Structural basis for the recognition of the SARS-CoV-2 by full-length human ACE2. Science. 2020.

2. Guan WJ, Ni ZY, Hu Y, Liang WH, Ou CQ, He JX, Liu L, Shan H, Lei CL, Hui D, et al. Clinical characteristics of coronavirus disease 2019 in China. N Engl J Med. 2020. PMID: 32109013.

3. Yang J, Zheng Y, Gou X, Pu K, Chen Z, Guo Q, Ji R, Wang H, Wang Y, Zhou Y, Prevalence of comorbidities in the novel Wuhan coronavirus (COVID-19) infection: a systematic review and metaanalysis, International Journal of Infectious Diseases

4. Toshiyuki Matsumoto, Ryoji Ozono, Tetsuya Oshima, Hideo Matsuura, Taijiro Sueda, Goro Kajiyama, Masayuki Kambe, Type 2 angiotensin II receptor is downregulated in cardiomyocytes of patients with heart failure, Cardiovascular Research, Volume 46, Issue 1, April 2000, Pages 73-81,

5. Juan Meng, Guohui Xiao, Juanjuan Zhang, Xing He, Min Ou, Jing Bi, Rongqing Yang, Wencheng Di, Zhaoqin Wang, Zigang Li, Hong Gao, Lei Liu \& Guoliang Zhang (2020) Renin-angiotensin system inhibitors improve the clinical outcomes of COVID-19 patients with hypertension, Emerging Microbes \& Infections, 9:1, 757-760, DOI: 10.1080/22221751.2020.1746200 